By

\author{
Antonio Desimone \\ and \\ Georg Dolzmann
}

IMA Preprint Series \# 2038

(April 2005)

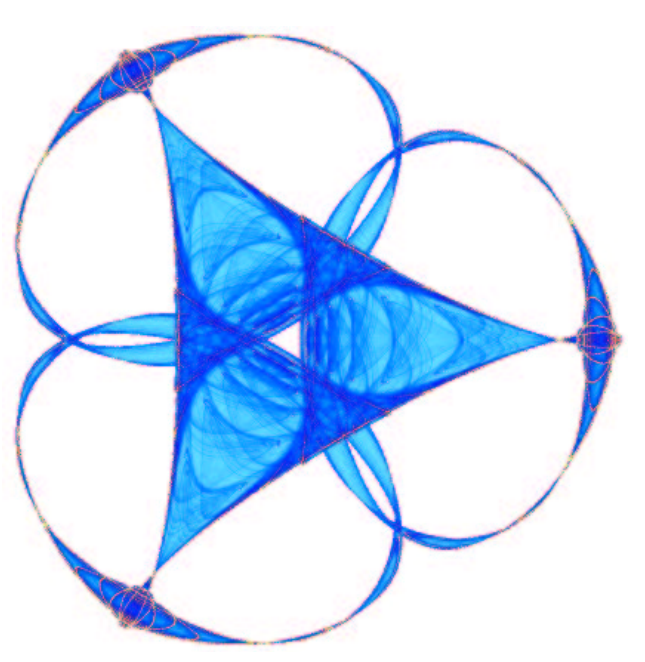

INSTITUTE FOR MATHEMATICS AND ITS APPLICATIONS

UNIVERSITY OF MINNESOTA

514 Vincent Hall

206 Church Street S.E.

Minneapolis, Minnesota 55455-0436

Phone: 612/624-6066 Fax: 612/626-7370

URL: http://www.ima.umn.edu 


\title{
STRIPE-DOMAINS IN NEMATIC ELASTOMERS: OLD AND NEW
}

\author{
ANTONIO DESIMONE* AND GEORG DOLZMANN ${ }^{\dagger}$
}

\begin{abstract}
Formation of stripe-domains has often been observed in nematic elastomers, starting from the pioneering work of Finkelmann and coworkers. One of the possible interpretations of this phenomenon is to view it as a material instability driven by energy minimization. This approach, first proposed by Warner and Terentjev, has been quite helpful in the analysis of stretching experiments of thin sheets, and in the modelling of soft elasticity associated with stripe-domain formation. Recently, complex stripe-domain patterns have been observed in nematic gels undergoing the isotropicto-nematic transition while being confined by two glass plates. We suggest that, once again, energy minimization can be seen as the driving mechanism for the formation of the observed patterns.
\end{abstract}

Key words. Polymers, Nematic elastomers, Domain patterns, Nonlinear elasticity, Nonconvex problems in the Calculus of Variations, Relaxation, Numerical simulation of microstructures.

AMS(MOS) subject classifications. 74N15, 74B20, $49 \mathrm{~J} 45$.

1. Introduction. Nematic elastomers consist of networks of crosslinked polymeric chains, each of which contains nematic rigid rod-like molecules (either as main-chain segments or as side attachments). These are called nematic mesogens: their tendency to align below a critical transition temperature promotes the formation of nematic order. Contrary to nematic liquids, however, the orientational degrees of freedom of the mesogens are coupled to the translational degrees of freedom of an underlying elastic solid (the rubbery polymer network). This coupling makes nematic elastomers very interesting as a model physical system, and it is also at the root of their technological interest as materials for actuators and for (mechanically tunable) optical devices. The recent monograph [1], and the extensive list of references cited therein give a comprehensive account of the history of the synthesis of liquid crystal elastomers, of the envisaged applications, and of the models proposed to explain their fascinating properties.

Nematic elastomers display interesting material instabilities. At high temperatures, the nematic mesogens are randomly oriented due to thermal fluctuations, and nematic elastomers behave like isotropic rubbers. Upon cooling through the nematic transition temperature, the nematic mesogens align causing a distorsion of the underlying rubber network. Imagine, for simplicity, the possibility that locally the mesogens are perfectly aligned at

\footnotetext{
${ }^{*}$ SISSA-International School for Advanced Studies, Via Beirut 2-4, 34014 Trieste, ITALY.

${ }^{\dagger}$ Department of Mathematics, University of Maryland, College Park, MD 20742, USA.
} 
the microscopic scale. Then symmetry dictates that the polymer network deform uniaxially, with the distinguished axis parallel to the common direction of the mesogens. Moving now to a more macroscopic scale, if the mesogens align along different directions in different parts of the sample, then different states of spontaneous distorsion may coexist within the same sample, generating patterns which are reminiscent of magnetic domains in ferromagnetic materials. Since the optical properties of the material are controlled by the orientation of the nematic mesogens, which changes from domain to domain, domain patterns are observable under polarized light. Indeed, regions where the mesogens are differently oriented may appear opaque or transparent when observed under crossed polarizers, depending on the relative orientation of mesogens and polarization of light.

The mechanical response of nematic elastomers to imposed deformation may be unusually soft, when compared to the response of ordinary rubber. The existence of domain patterns, and the ease with which they can evolve, is what allows nematic elastomers to respond to imposed macroscopic deformations with negligible internal stress, whenever the imposed strains may be accommodated by simply reorienting the nematic mesogens.

2. A minimalist model. Both the occurrence of domains, in a characteristic striped texture, and the existence of 'soft' deformation modes have been observed experimentally, in several different laboratories. These phenomena have also been analyzed in great detail by several groups, using a variety of different models. Many of these were represented at the IMA workshop [2], and we refer to the papers [3]-[8] for a sample of the many existing viewpoints on the subject. By contrast, we take here a minimalist approach, and proceed with the help of the most basic model we can think of. In essence, all what we do is to accept that the symmetry-breaking phase transformation associated with the establishment of nematic order is able to produce a number of distinct, symmetry-related, spontaneously deformed states, and that these states are the minima of a quadratic freeenergy density with the correct invariance properties. From here, we will try and deduce the largest possible number of rigorous consequences resulting from energy minimization, without further simplifications. Our aim is to contribute, in this way, to put into sharper focus the most generic and universal aspects of the phenomenon under study.

The first ingredient of our model is the spontaneous strain the material experiences when, starting from the isotropic parent phase, it undergoes the isotropic-to-nematic phase transformation. Assume that the nematic mesogens align, say, along the direction of a unit vector $\mathbf{n}$. Accepting the hypothesis of incompressibility, and assuming that the polymer chains stretch in the direction of $\mathbf{n}$, the resulting deformation is described by a uniaxial tensor of the form

$$
\mathbf{U}_{\mathbf{n}}:=a^{-1 / 3} \mathbf{n} \otimes \mathbf{n}+a^{1 / 6}(\mathrm{Id}-\mathbf{n} \otimes \mathbf{n})
$$


where $(\mathbf{n} \otimes \mathbf{n})_{i j}=\mathbf{n}_{i} \mathbf{n}_{j}$, Id is the identity, and $a<1$ is a material parameter quantifying how much a spherical random polymer coil spontaneously extends in the direction of $\mathbf{n}$ upon the alignment of the mesogens. Using frame-indifference and material symmetry (recall that the relevant symmetry is the one of the isotropic parent phase), we deduce from (2.1) that the set of spontaneous deformations has the form

$$
\mathbf{P} \mathbf{U}_{\mathbf{Q n}}=\mathbf{P Q} \mathbf{U}_{\mathbf{n}} \mathbf{Q}^{T}
$$

where $\mathbf{P}$ and $\mathbf{Q}$ are arbitrary rotations. A deformation gradient $\mathbf{F}$ belongs to the set (2.2) if and only if the ordered principal stretches $\lambda_{1}(\mathbf{F}) \leq$ $\lambda_{2}(\mathbf{F}) \leq \lambda_{3}(\mathbf{F})$ satisfy $\lambda_{1}(\mathbf{F})=\lambda_{2}(\mathbf{F})=a^{1 / 6}<1, \lambda_{3}(\mathbf{F})=a^{-1 / 3}>1$.

The next ingredient of our model is an energy penalization on deformation gradients $\mathbf{F}$ which are not in the set (2.2). The simplest choice for a quadratic, isotropic, frame-indifferent expression is a minor modification of the classical neo-hookean expression

$$
W(\mathbf{F})= \begin{cases}\mu\left(\lambda_{1}^{2}(\mathbf{F})+\lambda_{2}^{2}(\mathbf{F})+a \lambda_{3}^{2}(\mathbf{F})-3 a^{1 / 3}\right) & \text { if } \operatorname{det} \mathbf{F}=1 \\ +\infty & \text { else. }\end{cases}
$$

Here $\mu>0$ is a material parameter giving the rubber energy scale (defining the initial shear modulus of the material), while the constraint $\operatorname{det} \mathbf{F}=1$ on deformations with finite energy enforces incompressibility. Since the geometric mean of the squares of the principal stretches is not larger than the corresponding arithmetic mean, it is easy to see that (2.3) is always non-negative, and that it vanishes precisely on the set (2.2) (which we then call the set of the material's energy wells). The classical neo-hookean expression is simply obtained from (2.7) by setting $a=1$.

Finally, denoting by $\mathbf{y}$ the map that gives the deformed configuration of a body whose reference configuration is $\mathcal{B}$, and by $\nabla \mathbf{y}$ its gradient, the total energy reads

$$
E(\mathbf{y})=\int_{\mathcal{B}} W(\mathbf{y}(\mathbf{x})) d \mathbf{x} .
$$

We are interested in deformation maps which minimize this total energy when suitable loads or boundary displacements are prescribed.

Before proceeding with the analysis of our minimalist model, a few remarks are in order. Expression (2.3) can actually be derived from the one first proposed in [3] with the following procedure (see $[9,10])$. First, assume that nematic order is adequately described by a director field $\mathbf{x} \mapsto \mathbf{n}(\mathbf{x})$. Then perform an affine transformation of the spatial variables which corresponds to using, as reference configuration, the highly symmetric stressfree configuration of the isotropic parent phase. ${ }^{1}$ This leads to the following

\footnotetext{
${ }^{1}$ The reference configuration chosen in [3] is one of the stress-free configurations the material adopts after it has transformed to the nematic phase. One such configuration is the initial configuration in the stretching experiments discussed in Section 3.
} 
simple expression for the energy density, depending on the vector field $\mathbf{n}$ and on the tensor field $\mathbf{F}$

$$
W_{\mathrm{BTW}}(\mathbf{n}, \mathbf{F})=\mu\left(|\mathbf{F}|^{2}-(1-a)\left|\mathbf{F}^{T} \mathbf{n}\right|^{2}-3 a^{1 / 3}\right) .
$$

Finally, minimize the last expression with respect to $\mathbf{n}$ to obtain

$$
W(\mathbf{F})=\min _{|\mathbf{n}|=1} W_{\mathrm{BTW}}(\mathbf{n}, \mathbf{F}) .
$$

Notice that the $\mathbf{n}$ minimizing $W_{\mathrm{BTW}}$ is the eigenvector of $\mathbf{F}^{T} \mathbf{F}$ associated with its largest eigenvalue $\lambda_{3}^{2}(\mathbf{F})$. It follows that, in our simple-minded model, the nematic degrees of freedom are slaved to the elastic ones, and they are simply described by a director field which is aligned to the direction of the largest principal stretch at every point of the sample.

Moving to the analysis of our model, its most important feature is the non-convexity of energy density (2.3) for $a<1$. This implies that uniform configurations may have higher energy than complex domain patterns with the same average deformation. In fact, this is the mechanism proposed in [7] to explain the experimental observation that stretching a mono-domain sheet of nematic elastomer may induce spontaneous break-up into stripedomains [14].

The non-convexity of (2.3) also explains the experimentally observed soft deformation paths. They arise as energetically optimal fine phase mixtures, with volume fractions evolving with the applied loads. These fine phase mixtures effectively accomplish a convexification of the underlying rough energy landscape. A plateau in the stress-strain response is the precisely signature of a flat portion of the energy graph resulting from the convexification of the energy. We conclude that macroscopic soft deformation paths result, and should then be computable, from suitable convex hulls of the materials energy wells. In fact, using this idea, the characterization of all soft deformation paths associated with the set (2.2) was obtained in [10].

The existence of macroscopic deformations that can be resolved at no energy cost by microscopic mixtures of deformation gradients lying on the energy wells is only part of the story. One may ask what is, in general, the minimal energetic cost of imposing an arbitrary affine deformation $\mathbf{F}$ on the boundary $\partial \Omega$ of a representative volume $\Omega$, leaving the system free to develop fine structures in the interior of $\Omega$ whenever this is energetically advantageous. This is given by a well-defined mathematical object (see, e.g., [11]), the value at $\mathbf{F}$ of the quasi-convex envelope of the energy density $W$

$$
W_{\mathrm{qc}}(\mathbf{F}):=\inf _{\mathbf{y} \in \mathcal{A}}\left\{\frac{1}{|\Omega|} \int_{\Omega} W(\nabla \mathbf{y}(\mathbf{x})) d \mathbf{x}: \mathbf{y}(\mathbf{x})=\mathbf{F} \mathbf{x} \text { on } \partial \Omega\right\}
$$


where $|\Omega|$ is the volume of $\Omega$ and the infimum is taken over the set $\mathcal{A}$ of Lipschitz-continuous maps such that $\operatorname{det} \nabla \mathbf{y}(\mathbf{x}) \equiv 1$. It can be shown that the right-hand-side of (2.7) does not depend on the geometry of $\Omega$.

An explicit formula for the quasi-convex envelope of (2.3) has been derived in [12]. For volume-preserving deformation gradients it reads

$$
W_{\mathrm{qc}}(\mathbf{F})= \begin{cases}0 & \left(\text { phase L) if } \lambda_{1} \geq a^{1 / 6}\right. \\ W(\mathbf{F}) & \left(\text { phase S) if } a^{1 / 2} \lambda_{3}^{2} \lambda_{1}>1\right. \\ \mu\left(\lambda_{1}^{2}+2 a^{1 / 2} \lambda_{1}^{-1}-3 a^{1 / 3}\right) & (\text { phase I) else }\end{cases}
$$

while $W_{\mathrm{qc}}(\mathbf{F})=+\infty$ if $\operatorname{det} \mathbf{F} \neq 1$. Here the labels L, S, and I refer to the fact that the resulting material response is liquid-like, solid-like, or of an intermediate type, see the discussion below.

The formula above gives a very precise picture of the macroscopic mechanical response resulting from our model, and of its microscopic origin. There are three regimes in (2.8), arising from the collective behavior of energetically optimal fine phase mixtures. They represent three different modes of macroscopic mechanical response, and they correspond to three different patterns of microscopic decomposition of the macroscopic deformation gradient $\mathbf{F}$. Phase $\mathrm{L}$ describes a liquid-like response (at least within the ideally soft approximation embedded in the expression (2.3) for the microscopic energy density; the semi-soft case is discussed in [13]). All gradients falling in this region of the phase diagram can be sustained at zero internal stress (in other words, the zero level set of $W_{q c}$ is the set of all soft deformation paths mentioned above). To resolve microscopically the whole of phase L (in particular, to resolve the deformation gradient $\mathbf{F}=\mathrm{Id}$ ) it is necessary to allow for relatively complex microstructures (layers-within-layers, see the right panel in Figure 1). Phase S describes a solid-like response in which fine phase mixtures are ruled out. As a consequence, in this regime the effective macroscopic energy $W_{q c}$ reproduces the microscopic energy $W$ with no changes. Finally, gradients in the intermediate phase I can transmit stresses (unlike phase L) through microstructure formation (unlike phase $\mathrm{S}$ ). The microscopic patterns required to resolve phase I have a relatively simple geometry (laminates, or simple-layers, see the left panel in Figure 1). Patterns of this kind have been frequently observed experimentally after being first reported in [14]. The first attempt to explain them through elastic energy minimization is in [7].

By decoupling the physical length scales into microscopic ones (which determine the effective energetics but are averaged out in the kinematics) and macroscopic ones (e.g., those which determine the deformed shape of a sample in a stretching experiment), expression (2.8) leads to a coarsegrained version of our model. In order to resolve macroscopic quantities, we minimize the effective energy

$$
E_{\text {eff }}(\mathbf{y})=\int_{\mathcal{B}} W_{\mathrm{qc}}(\mathbf{y}(\mathbf{x})) d \mathbf{x}
$$




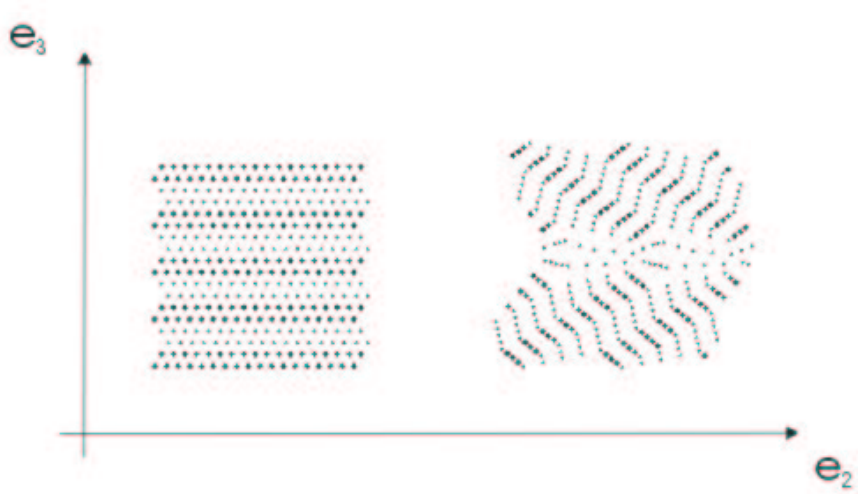

FIG. 1. Geometry of energetically optimal microstructures: simple-layers (left) and layers-within-layers (right). The light and dark dots hint at the optical contrast these microstructure would produce in nematic elastomers under polarized light (adapted from [9]).

subject to the boundary conditions that are relevant to the loading experiment we want to model. This can be done numerically, giving a field $\mathbf{x} \mapsto \mathbf{F}(\mathbf{x})$. Then, by locating the computed macroscopic deformation within one of the three phases L, S, I of the phase diagram, we can reconstruct, from the field of computed local deformation gradients $\mathbf{x} \mapsto \mathbf{F}(\mathbf{x})$, the corresponding field of local (energy minimizing) microstructures.

Before closing this section, one word about the length scales of the domain patterns. Energy (2.2) does not contain an intrinsic length scale. Thus, the minimization procedure (2.7) leading to $W_{\mathrm{qc}}$ can lead to domain patterns which are infinitely fine with respect to the size of a representative volume element, and which manifest themselves as (infinitely refining) sequences of deformation patterns driving the energy to its infimum. Clearly, in reality, physical mechanisms not active in our simple--minded model do establish a smallest characteristic length scale. Whenever the size of the sample of interest is large compared to this characteristic length, our approach can be used to gather detailed information on the macroscopic response of the sample (e.g., the force-stretch curve), and gross information on the domain patterns responsible for the macroscopic behavior. More detailed information on domain patterns requires explicit resolution of length scales. One natural possibility is to consider models containing higher order gradients (e.g., Frank-type terms in the gradient of the director, see e.g. [4]). Needless to say, since the corresponding numerical simulations will need to resolve much finer spatial scales (typically, at the sub-micron scale), a substantial increase of the computational cost for obtaining a force-stretch diagram for, say, a mm-sized sample should be expected. 
3. Stripe-domain patterns: the classics. The computational approach outlined in the previous section, and based on the expression (2.7) for the energy density, has been used in [15] for the numerical simulation of stretching experiments of sheets of nematic elastomer held between two rigid clamps. The simulations are designed to reproduce the classical experimental setting of Kundler and Finkelmann [14], where stripe-domain patterns were first observed.

The specimen is a thin sheet of nematic elastomer. We choose a reference frame with axis $x_{1}$ parallel to the thickness direction. Moreover, we assume that the specimen is prepared with the director uniformly aligned along $x_{3}$, and is then stretched along $x_{2}$. By reorienting the director from the $x_{3}$ to the $x_{2}$ direction, the material can accommodate the imposed stretches without storing elastic energy. As it is well known, see e.g. [1], a uniform rotation of the director would induce large shears, which are incompatible with the presence of the clamps. Director reorientation occurs instead with the development of spatial modulations shaped as bands parallel to the $x_{2}$ axis. This is the origin of the striped texture observed in the experiments.

The numerical simulations allow us to analyze the stretching experiments in more detail. If the clamps do not allow lateral contraction, the reorientation of the director towards the direction of the imposed stretch is severely hindered. This constraint is stronger near the clamps, and it decays away from them producing two interesting effects. On one hand, the induced microstructures are spatially inhomogeneous, with director reorientation occurring more rapidly in the regions far away from the clamps. On the other hand, the stress-strain response shows a marked dependence on the geometry of the sample, with the influence of the clamps becoming less pronounced as the aspect ratio length/width increases. These effects are documented in Figure 2 and Figure 3, which show good qualitative agreement with both the experimental results from the Cavendish Laboratories [1], and with the X-ray scattering measurements in [16].

The stripe domain patterns appearing in Figure 3 are all simple laminates, either in phase $\mathrm{L}$ or in phase I. Focusing on the point at the center of the sample (the bottom left corner in the plots of the deformed shape), the material is in phase $\mathrm{L}$ as long as no force is transmitted at the clamps. The computed deformation gradient is

$$
\mathbf{F}_{\lambda}=\left(\begin{array}{ccc}
a^{1 / 6} & 0 & 0 \\
0 & \lambda & 0 \\
0 & 0 & a^{-1 / 6} / \lambda
\end{array}\right)
$$



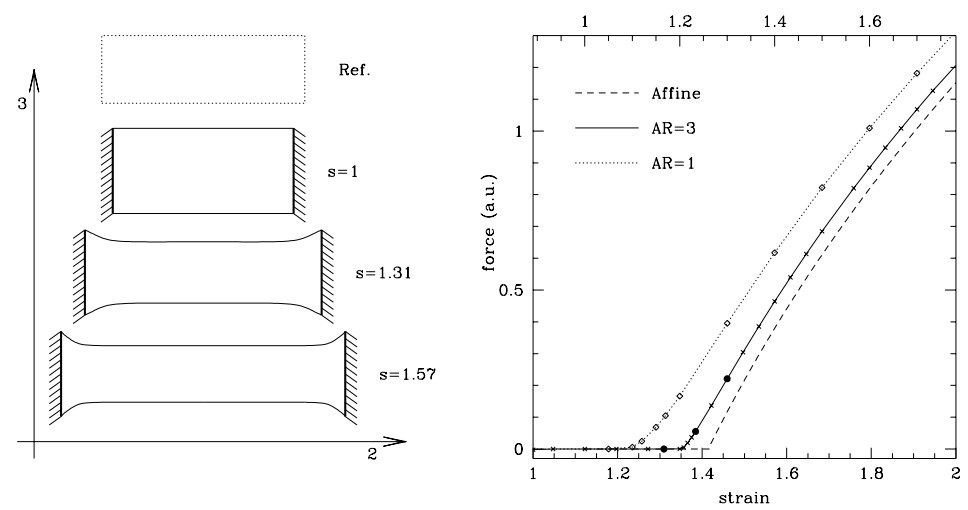

FIG. 2. Numerical simulation of stretching experiments on thin sheets of nematic elastomers: geometry (left) and force-stretch diagrams for several aspect ratios AR (right). The panel on the left shows four configurations, namely, reference, initial, and the two at stretches $s=1.31$ and $s=1.57$ for the geometry with $A R=3$. On the corresponding force-stretch curve on the right panel, full dots mark the representative points of configurations shown in Figure 3 (adapted from [15]).
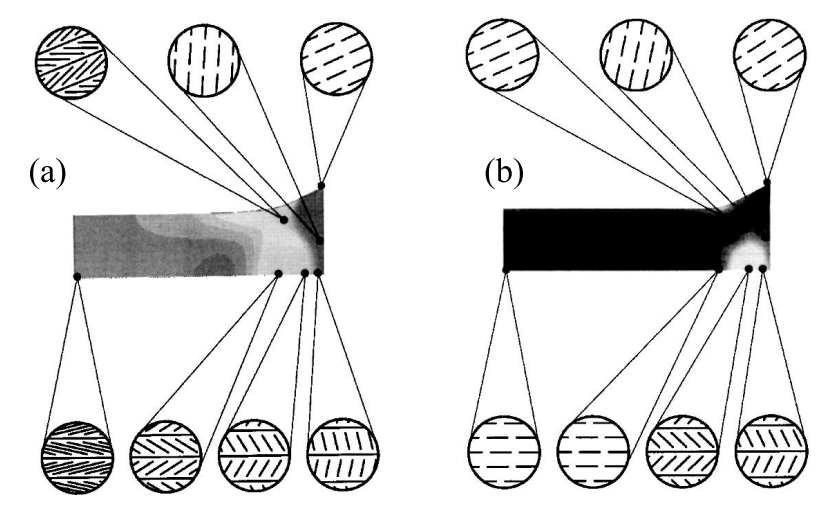

FIG. 3. Numerical simulation of stretching experiments on thin sheets of nematic elastomers, based on the coarse-grained energy $W_{\mathrm{qc}}$, at stretches $s=1.31$ (a), and $s=1.38$ (b). Only one-quarter of the sample is shown since the rest of the solution can be obtained by symmetry. The circular insets display energetically optimal microstructures at some selected locations within the sample. The sticks give the local orientation of the principal direction of maximal stretch, i.e., the orientation of the nematic director (adapted from [15]).

with $\lambda$ varying from $a^{1 / 6}$ to $a^{-1 / 3}$. This is resolved by a simple laminate in which the deformation gradient oscillates between the values

$$
\mathbf{F}_{\lambda}^{ \pm}=\left(\begin{array}{ccc}
a^{1 / 6} & 0 & 0 \\
0 & \lambda & \pm \delta \\
0 & 0 & a^{-1 / 6} / \lambda
\end{array}\right)
$$


in stripes perpendicular to $x_{3}$, i.e., with a geometry similar to the one shown in the left panel of Figure 1. The value of $\delta=\delta(\lambda)$ is obtained from $\delta^{2}=\left(a^{-2 / 3}-\lambda^{2}\right)\left(1-a^{1 / 3} \lambda^{-2}\right)$, which ensures that $\mathbf{F}_{\lambda}^{ \pm}$has the characteristic principal stretches giving $W\left(\mathbf{F}_{\lambda}^{ \pm}\right)=0$. Notice that the kinematic compatibility condition $\mathbf{F}_{\lambda}^{+}-\mathbf{F}_{\lambda}^{-}=\mathbf{a} \otimes \mathbf{N}$, where $\mathbf{N}$ is the reference normal to the stripes and $\mathbf{a}$ is a shear vector, is satisfied with $\mathbf{a}=2 \delta(\lambda) \mathbf{e}_{2}$ and $\mathbf{N}=\mathbf{e}_{3}$. This guarantees the existence of a continuous map $\mathbf{y}$ such that $\nabla \mathbf{y}(\mathbf{x})=\mathbf{F}_{\lambda}^{ \pm}$in layers with normal $\mathbf{e}_{3}$.

Force starts being transmitted through the sample when the deformation gradient in the central point moves to the region I of the phase diagram. The computed deformation gradient is now of the form

$$
\mathbf{F}_{\lambda_{1}}=\left(\begin{array}{ccc}
\lambda_{1} & 0 & 0 \\
0 & 1 / \lambda_{1} \lambda_{3} & 0 \\
0 & 0 & \lambda_{3}
\end{array}\right)
$$

where $\lambda_{3}>a^{-1 / 3}$ forces $\lambda_{1}<a^{1 / 6}$. This is resolved by simple laminates similar to the ones above. The deformation gradient oscillates between the values

$$
\mathbf{F}_{\lambda_{1}}^{ \pm}=\left(\begin{array}{ccc}
\lambda_{1} & 0 & 0 \\
0 & 1 / \lambda_{1} \lambda_{3} & \pm \delta \\
0 & 0 & \lambda_{3}
\end{array}\right)
$$

in stripes perpendicular to $x_{3}$, and $\delta=\delta\left(\lambda_{1}\right)$ is computed by requiring that the principal stretches be those giving the minimal energy at given $\lambda_{1}$, namely, $\left(\lambda_{1}, a^{1 / 4} \lambda_{1}^{-1 / 2}, a^{-1 / 4} \lambda_{1}^{-1 / 2}\right)$, see [15].

4. Stripe-domain patterns: recent observations. The setup of the stretching experiments described in the previous section has one peculiarity. In the initial configuration, the film thickness near the center of the sample is $a^{1 / 6}$ times the thickness in the reference configuration. As the film is stretched, the film thickness either stays unchanged (this is the regime given by (3.1), and it represents an unusual behavior when compared to that of a conventional rubber) or it decreases (this is the regime given by (3.3)). This implies that the smallest principal stretch can never exceed the value $a^{1 / 6}$. As a consequence microstructures of the layerswithin-layers type are not accessible in the kind of stretching experiments described above, no matter how close the material is to the limit of ideal softness.

In the course of the workshop [2], the following recent experiment by Meyer and Meng was presented. A thin film of a soft nematic gel, confined between two horizontal glass plates, is cooled through the isotropic-tonematic transition temperature $T_{I N}$ while its director is kept vertical by an applied electric field. When the external field is removed, the nematic director is no longer frozen, and in-plane stripe domains develop and become visible through the glass plates. 
If one focuses on points far away from the sample edges, one may think of the glass plates as infinitely extended so that, by symmetry, all the in-plane stretches are principal stretches with the same value. The constraining action of the glass plates, which do not allow the film thickness to expand, coupled with the incompressibility constraint, then results in imposing that the material does not deform at all, or

$$
\mathbf{F}=\mathrm{Id}
$$

at least in average, and far away from the lateral edges. This macroscopic deformation gradient can be resolved by the spontaneous deformations of the set (2.2).

To discuss the experiment more quantitatively, we fix a reference frame with axes $x_{1}$ and $x_{2}$ parallel to the mid-surface of the film, and axis $x_{3}$ along the thickness direction. The geometry of the energetically optimal microstructures resolving (4.1) entails two orders of lamination. One is along the film thickness, to accommodate the fact that at temperatures below $T_{I N}$, the natural thickness associated with a vertically oriented director is larger than the distance between the two glass plates. The second lamination, in the plane of the film, is the one that should be responsible for the observed contrast. Were this second order of lamination absent or, said differently, were the director to buckle while remaining in one plane (say, the $x_{1} x_{3}$ plane), then the film would have to contract (by the amount $\left.a^{1 / 6}<1\right)$ in the direction of $x_{2}$. Since this contraction is incompatible with the constraints introduced by the experimental apparatus, in-plane stripe-domains are generated.

To test this hypothesis, we looked for the possibility of resolving (4.1) with exactly four deformation gradients lying in the set of zero energy spontaneous distorsions (2.2). The geometry of the construction is sketched in Figure 4. Notice that, in the layers-within-layers construction of Figure 1, fine layers are nested inside coarser layers and kinematic compatibility across the interfaces of the coarse layers holds only approximately. By contrast, in Figure 4, kinematic compatibility across all interfaces is satisfied exactly, see formulas (4.2)-(4.5) below. More in detail, we set $\eta=a^{-1 / 6}$ and consider the following deformation gradients

$$
\begin{aligned}
& \mathbf{F}_{11}=\left(\begin{array}{ccc}
1 & 0 & \frac{1}{\eta}-\eta \\
\eta-\frac{1}{\eta} & 1 & 1-\eta^{2} \\
0 & 0 & 1
\end{array}\right) \quad \mathbf{F}_{22}=\left(\begin{array}{ccc}
1 & 0 & \frac{1}{\eta}-\eta \\
-\eta+\frac{1}{\eta} & 1 & \eta^{2}-1 \\
0 & 0 & 1
\end{array}\right) \\
& \mathbf{F}_{12}=\left(\begin{array}{ccc}
1 & 0 & \eta-\frac{1}{\eta} \\
\eta-\frac{1}{\eta} & 1 & \eta^{2}-1 \\
0 & 0 & 1
\end{array}\right) \quad \mathbf{F}_{21}=\left(\begin{array}{ccc}
1 & 0 & \eta-\frac{1}{\eta} \\
-\eta+\frac{1}{\eta} & 1 & 1-\eta^{2} \\
0 & 0 & 1
\end{array}\right) \text {. }
\end{aligned}
$$



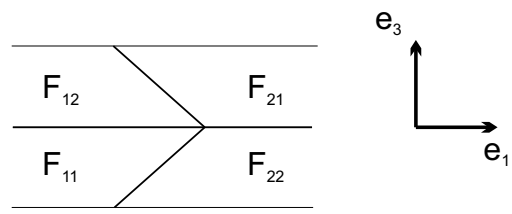

FIG. 4. Geometry of our zero-energy four gradient construction: reference (left) and deformed (right) configurations for $\eta=1.1225$ corresponding to $a=0.5$.

Clearly,

$$
\begin{aligned}
& \mathbf{F}_{11}-\mathbf{F}_{12}=-2\left(\eta-\frac{1}{\eta}, \eta^{2}-1,1\right) \otimes \mathbf{e}_{3} \\
& \mathbf{F}_{22}-\mathbf{F}_{21}=-2\left(\eta-\frac{1}{\eta}, 1-\eta^{2}, 1\right) \otimes \mathbf{e}_{3}
\end{aligned}
$$

so that interfaces between $\mathbf{F}_{11}$ and $\mathbf{F}_{12}$, and between $\mathbf{F}_{22}$ and $\mathbf{F}_{21}$ are possible, with normal parallel to $\mathbf{e}_{3}$ in the reference configuration. Moreover,

$$
\begin{aligned}
& \mathbf{F}_{21}-\mathbf{F}_{12}=-2\left(\eta-\frac{1}{\eta}\right) \mathbf{e}_{2} \otimes(1,0, \eta) \\
& \mathbf{F}_{22}-\mathbf{F}_{11}=-2\left(\eta-\frac{1}{\eta}\right) \mathbf{e}_{2} \otimes(1,0,-\eta)
\end{aligned}
$$

so that an interface between $\mathbf{F}_{21}$ and $\mathbf{F}_{12}$ is possible with reference normal parallel to $(1,0, \eta)$, and an interface between $\mathbf{F}_{22}$ and $\mathbf{F}_{11}$ is possible with reference normal parallel to $(1,0,-\eta)$.

We now plot the deformed configuration of the film, see Figure 4 and Figure 5, using the value $\eta=1.1225$ corresponding to $a=0.5$. For this purpose, it is useful to compute the deformed orientation $\mathbf{F}^{*} \mathbf{N}$ of each layer normal $\mathbf{N}$, where $\mathbf{F}^{*}=(\operatorname{det} \mathbf{F}) \mathbf{F}^{-T}$ is the cofactor of the deformation gradient inside the layer. Since

$$
\begin{aligned}
\mathbf{F}_{11}^{*}=\left(\begin{array}{ccc}
1 & -\xi & 0 \\
0 & 1 & 0 \\
\xi & -\Lambda & 1
\end{array}\right) & \mathbf{F}_{22}^{*}=\left(\begin{array}{ccc}
1 & \xi & 0 \\
0 & 1 & 0 \\
\xi & \Lambda & 1
\end{array}\right) \\
\mathbf{F}_{12}^{*}=\left(\begin{array}{ccc}
1 & -\xi & 0 \\
0 & 1 & 0 \\
-\xi & \Lambda & 1
\end{array}\right) & \mathbf{F}_{21}^{*}=\left(\begin{array}{ccc}
1 & \xi & 0 \\
0 & 1 & 0 \\
-\xi & -\Lambda & 1
\end{array}\right)
\end{aligned}
$$

where $\xi=\eta-1 / \eta$ and $\Lambda=1-\eta^{2}+(\eta-1 / \eta)^{2}$, we have

$$
\begin{aligned}
\mathbf{F}_{11}^{*} \mathbf{e}_{3} & =\mathbf{F}_{12}^{*} \mathbf{e}_{3}=\mathbf{F}_{21}^{*} \mathbf{e}_{3}=\mathbf{F}_{22}^{*} \mathbf{e}_{3}=\mathbf{e}_{3}, \\
\mathbf{F}_{12}^{*}(1,0, \eta) & =\mathbf{F}_{21}^{*}(1,0, \eta)=\left(1,0, \frac{1}{\eta}\right),
\end{aligned}
$$




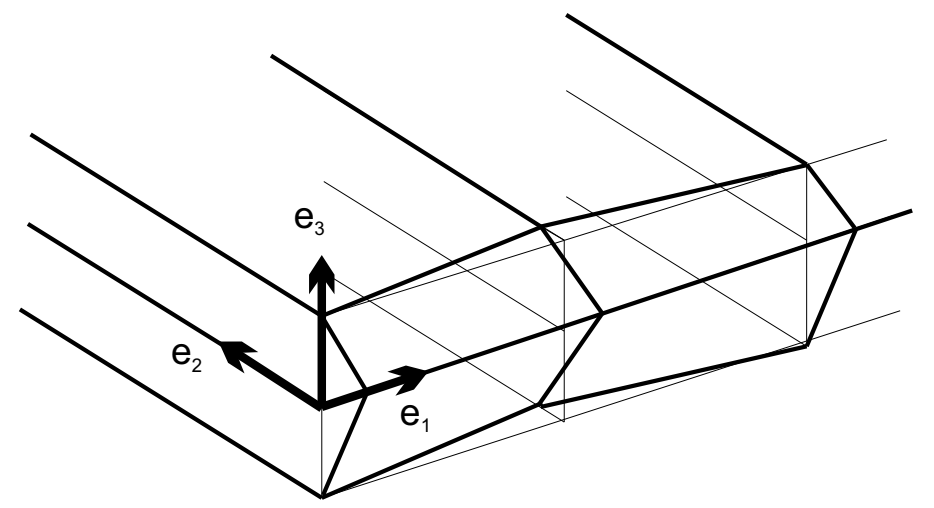

FIG. 5. Sketch of a domain pattern resolving $\mathbf{F}=\mathrm{Id}$. This models the possible behavior of a film undergoing the isotropic-nematic phase transformation while being constrained between two parallel plates.

and

$$
\mathbf{F}_{11}^{*}(1,0,-\eta)=\mathbf{F}_{22}^{*}(1,0,-\eta)=\left(1,0,-\frac{1}{\eta}\right)
$$

For what concerns the displacement of material particles, notice that the relative motion of the layer deformed according to $\mathbf{F}_{22}$ (respectively, $\mathbf{F}_{21}$ ) relative to that deformed according to $\mathbf{F}_{11}$ (respectively, $\mathbf{F}_{12}$ ) is a shear of amplitude $2 \sqrt{1+\eta^{2}}(\eta-1 / \eta)$ in the $\mathbf{e}_{2}$ direction. This follows from formulas (4.4) and (4.5), by normalizing the modulus of the interface normals $(1,0, \pm \eta)$ to one. The resulting deformation is sketched in Figure 5, where attention should be paid to the alternating shears in the plane $x_{1} x_{2}$ which are responsible for the observed stripe pattern.

Finally, we give the orientations of the nematic director implied by our four-gradient construction. As noted above, within our model, the nematic director is always aligned with the direction of maximal stretch. This implies that, in the deformed configuration, the orientation $\overline{\mathbf{n}}$ of the director will be that of the eigenvector of $\mathbf{F F}^{T}$ associated with its largest eigenvalue $\lambda_{3}^{2}(\mathbf{F})$. We thus obtain the following relations of proportionality

$$
\begin{aligned}
& \overline{\mathbf{n}}_{11} \propto\left(\begin{array}{c}
-\eta \\
-\eta^{2} \\
1
\end{array}\right) \quad \overline{\mathbf{n}}_{22} \propto\left(\begin{array}{c}
-\eta \\
\eta^{2} \\
1
\end{array}\right) \\
& \overline{\mathbf{n}}_{12} \propto\left(\begin{array}{c}
\eta \\
\eta^{2} \\
1
\end{array}\right)
\end{aligned}
$$

A more detailed analysis of zero energy deformation patterns compatible with (4.1) and, more generally, of the stripe patterns that may arise 
within the confined geometry described at the beginning of this section will appear elsewhere [17].

5. Conclusions and Outlook. Nematic elastomers have been synthesized relatively recently [18]-[21]. Nevertheless, they have already attracted considerable attention in the Chemistry, Engineering, Mathematics, and Physics literature. The same is true, in particular, for the stripedomain patterns they exhibit.

Our interest in nematic elastomers arose from the realization that the symmetry-breaking isotropic-to-nematic phase transformation which is at the root of their fascinating material instabilities has close analogies with the martensitic phase transformations exhibited by shape-memory alloys. While in the latter case, however, the underlying material symmetry is the discrete crystallographic symmetry of the austenitic parent phase, in the case of nematic elastomers the full isotropic symmetry of the high temperature amorphous polymer is available. It soon became apparent that the mathematical techniques developed for the study of displacive phase transformations in crystals are applicable to a radically different class of systems (polymers, rather than crystals) and that the simplifications accompanying the enhanced material symmetries lead to results of unprecedented completeness. One such result is the development of a combined analytical-computational approach in which the original problem is first simplified with the use of mathematical analysis, and then attacked computationally. As described above, this approach has been used with some success to simulate numerically stretching experiments of thin sheets of nematic elastomers. We believe that this combination of analysis and computation has a great potential in shedding further light on the mechanical response of nematic elastomers and, more generally, of all systems whose mechanical response is microstructure-driven.

The analogy between shape memory alloys and nematic elastomers as shape-memory polymers is fruitful. It reveals to us the underlying structure, hence the ultimate simplicity of seemingly complicated stripe-domain patterns. And yet, trivially, analogy is not identity. The devil (just like system specificity coming from the different underlying physical mechanisms) is in the details. Examples are the detailed structure of a wall between two adjacent domains, or the geometric details of how two differently oriented systems of stripe-domains meet, or the time scales with which domains appear, disappear, and respond to external fields and loads. It is through such details that the complexity of these seemingly simple domain patterns really emerges. And we should be prepared to use sharper (but, unavoidably, more complicated and system specific) models to understand them.

Acknowledgments. This paper draws on an ongoing collaboration with S. Conti (University of Duisburg-Essen) and it was written during visits of both authors to the IMA, and of the second author to SISSA. 
The financial support of both Institutions is gratefully acknowledged. GD acknowledges also support through the NSF via grant DMS-0405853.

\section{REFERENCES}

[1] Warner M. and Terentjev E.M., Liquid Crystal Elastomers, Clarendon Press, Oxford 2003.

[2] IMA Workshop on Modeling of Soft Matter, University of Minnesota, Minneaplis, September 2004.

[3] Bladon P., Terentjev E.M., and Warner M., 1993. Transitions and instabilities in liquid-crystal elastomers. Phys. Rev. E 47: R3838-R3840.

[4] Finkelmann H., Kundler I., Terentuev E.M., and Warner M., 1997. Critical stripe-domain instability of nematic elastomers. J. Phys. II France 7: 1059-1069.

[5] Fried E. And Korchagin V., 2002. Striping of nematic elastomers. Int. J. Solids Structures 39: 3451-3467.

[6] Martinoty P., Stein P., Finkelmann H., Pleiner H., and Brand H.R., 2004. Mechanical properties of monodomain side chain nematic elastomers, Eur. Phys. J. E 14: 311.

[7] Verwey G.C., Warner M., And Terentjev E.M., 1996. Elastic instability and stripe domains in liquid crystalline elastomers. J. Phys. II France 6: $1273-1290$.

[8] Weilepp J. and Brand H., 1996. Director reorientation in nematic-liquis-singlecrystal elastomers by external mechanical stress. Europhys. Lett. 34: 495-500.

[9] DeSimone A., 1999. Energetics of fine domain structures. Ferroelectrics 222: $275-284$.

[10] DeSimone A. And Dolzmann G., 2000. Material instabilities in nematic elastomers. Physica D 136: 175-191.

[11] Müller S., 1999. Variational models for microstructure and phase transitions. In: Bethuel F., Huisken G., Müller S., Steffen K., Hildebrandt S., Struwe M. (Eds.), Calculus of Variations and Geometric Evolution Problems, Lectures given at the 2nd Session of the Centro Internazionale Matematico Estivo, Cetraro 1996. Springer, Berlin.

[12] DeSimone A. And Dolzmann G., 2002. Macroscopic response of nematic elastomers via relaxation of a class of $\mathrm{SO}(3)$-invariant energies. Arch. Rat. Mech. Anal. 161: 181-204.

[13] Conti S., DeSimone A., and Dolzmann G., 2002. Semi-soft elasticity and director reorientation in stretched sheets of nematic elastomers. Phys. Rev. E 60: 61710-1-8.

[14] Kundler I. And Finkelmann H., 1995. Strain-induced director reorientation in nematic liquid single crystal elastomers. Macromol. Rapid Comm. 16: $679-686$

[15] Conti S., DeSimone A., and Dolzmann G., 2002. Soft elastic response of stretched sheets of nematic elastomers: a numerical study. J. Mech. Phys. Solids 50: 1431-1451.

[16] Zubarev E.R., Kuptsov S.A., Yuranova T.I., Talroze R.V., and Finkelmann H., 1999. Monodomain liquid crystalline networks: reorientation mechanism from uniform to stripe domains. Liquid Crystals 26: 1531-1540.

[17] DeSimone A. And Dolzmann G., 2005. In preparation.

[18] Finkelmann H., and Rehage G., 1984. Liquid crystal side chain polymers. Adv. Polymer Sci. 60/61: 99.

[19] Finkelmann H., Gleim W., Kock H.J., and Rehage G., 1985. Liquid crystalline polymer network - Rubber elastic material with exceptional properties. Makromol. Chem. Suppl. 12: 49. 
[20] Küpfer J. and Finkelmann H., 1991. Nematic liquid single-crystal elastomers. Makromol. Chem. Rapid Comm. 12: 717-726.

[21] Zentel R., 1989. Liquid crystal elastomers. Angew. Chem. Adv. Mater. 101: 1437. 